\title{
Aplicación de lixiviados de vermicompost y respuesta agronómica de dos variedades de pimiento
}

\author{
Application of vermicompost leakage and agronomic response of two \\ varieties of pepper
}

Alcívar LlM, Vera R JH, Arévalo SO, Arévalo SB, Pachar OL, Castillo RC, et al. Aplicación de lixiviados de vermicompost y respuesta agronómica de dos variedades de pimiento. Rev Colombiana Cienc Anim. Recia. 2021; 13(1):e793. https://doi.org/10.24188/recia.v13.n1.2021.793

Universidad de Sucre, Colombia

Los autores permiten a RECIA reimprimir el material publicado en él. En caso de que un autor quiera traducir o usar una publicación parcial o completa de nuestro Diario, el autor debe obtener un permiso por escrito del editor de la revista.

Copyright (C) 2021. El (los) autor (es). Este es un artículo de acceso abierto distribuido bajo los términos de Creative Commons Attribution 4.0 (https://creativecommons.org/licenses/by-ncsa/4.0/), El uso, distribución o reproducción está permitido, siempre que se acrediten al autor original y al propietario del copyright y que se cite la publicación original en esta revista, de acuerdo con la práctica académica aceptada. No se permite el uso, distribución o reproducción que no cumpla con estos términos. 


\title{
Aplicación de lixiviados de vermicompost y respuesta agronómica de dos variedades de pimiento
}

\author{
Application of vermicompost leakage and agronomic response of two varieties of \\ pepper
}

María Fernanda Alcívar Llivicura. M.Sc;

Instituto Superior Tecnológico Enrique Noboa. Carrera Tecnología en

DOI: https://doi.org/10.24188/recia.v13.n1.2021.793

Producción Agropecuaria. La Troncal, Cañar, Ecuador.

*maryfer2323@hotmail.com

(D) http://orcid.org/0000-0001-9222-3436

José Humberto Vera Rodríguez. M.Sc.

Instituto Superior Tecnológico Enrique Noboa. Carrera Tecnología en

Producción Agropecuaria. La Troncal, Cañar, Ecuador.

humbertorichi@hotmail.com

(i) https://orcid.org/0000-0003-3027-059X

Omar Juvenal Arévalo Serrano. Tnglo.

Instituto Superior Tecnológico Enrique Noboa. Carrera Tecnología en

Producción Agropecuaria. La Troncal, Cañar, Ecuador.

omararevalo364@gmail.com

(D) https://orcid.org/0000-0003-3031-8581

Byron David Arévalo S. Tnglo.

Instituto Superior Tecnológico Enrique Noboa. Carrera Tecnología en

Producción Agropecuaria. La Troncal, Cañar, Ecuador.

byronarevalo325@gmail.com

(D) https://orcid.org/0000-0002-3222-6034

Lorena Elizabeth Pachar 0. Tnglo.

Instituto Superior Tecnológico Enrique Noboa. Carrera Tecnología en Producción Agropecuaria. La Troncal, Cañar, Ecuador.

lore.pachar2198@gmail.com

(iD) https://orcid.org/0000-0001-7586-8267

Charly Bryan Castillo R. Tnglo.

Instituto Superior Tecnológico Enrique Noboa. Carrera Tecnología en Producción Agropecuaria. La Troncal, Cañar, Ecuador.

castillobrowm@gmail.com

(D) https://orcid.org/0000-0002-4493-1937

Lisbeth Katherine Carlosama M. Tnglo.

Instituto Superior Tecnológico Enrique Noboa. Carrera Tecnología en Producción Agropecuaria. La Troncal, Cañar, Ecuador.

carlosamamorakata@gmail.com

(D) https://orcid.org/0000-0003-3233-389X

Joan Andrés Arizabal C. Tnglo.

Instituto Superior Tecnológico Enrique Noboa. Carrera Tecnología en

Producción Agropecuaria. La Troncal, Cañar, Ecuador.

joanarizabal48@gmail.com

(D) https://orcid.org/0000-0002-7161-1445

Noé David Paltán M. Tnglo.

Instituto Superior Tecnológico Enrique Noboa. Carrera Tecnología en

Producción Agropecuaria. La Troncal, Cañar, Ecuador.

noepaltan20@gmail.com

(D) https://orcid.org/0000-0001-7874-1130

Recepción: Julio 2020

Aprobación: Marzo 2021

Publicación: Mayo 2021

Como citar (Vancouver).

Alcívar LIM, Vera R JH, Arévalo SO, Arévalo SB, Pachar OL, Castillo RC, et al. Aplicación de lixiviados de vermicompost y respuesta agronómica de dos variedades de pimiento. Rev Colombiana Cienc Anim. Recia. 2021; 13(1):e793. https://doi.org/10.24188/recia.v13.n1.2021.793 


\title{
RESUMEN
}

El objetivo del presente trabajo fue evaluar el efecto de lixiviados de vermicompost a base de Residuos Vegetales y Gallinaza; Residuos Vegetales y Agroindustriales y la combinación de los dos lixiviados, en dosis de 60 y $90 \mathrm{~L} \mathrm{ha}^{-1}$ sobre el desarrollo y producción del pimiento. El estudió se efectuó en variedades de pimiento Marconi y Wonder, en total se establecieron siete tratamientos con tres repeticiones. La mejor respuesta en diámetro del fruto en ambas variedades evaluadas correspondió a los tratamientos de Lixiviado de Residuos Vegetales y Agroindustriales $90 \mathrm{~L} \mathrm{ha}^{-1}$ y Lixiviado Combinado $90 \mathrm{~L} \mathrm{ha}^{-1}$ siendo 52 y $15 \%$ superiores al control, respectivamente. Por otro lado, la mayor longitud del fruto fue evidenciada en el tratamiento Lixiviado Combinado $90 \mathrm{~L} \mathrm{ha}^{-1}$. En tanto que, la biomasa radicular incrementó en todos los tratamientos donde se aplicó el lixiviado. En la producción, definida por peso del fruto, los tratamientos sobresalientes fueron Lixiviado de Residuos Vegetales y Agroindustriales $90 \mathrm{~L} \mathrm{ha}^{-1}$ y Lixiviado Combinado $90 \mathrm{~L} \mathrm{ha}^{-1}$. De este modo, podemos concluir que el uso de lixiviados de vermicompost en dosis de $90 \mathrm{~L} \mathrm{ha}^{-1}$ representa una alternativa para potenciar el rendimiento del pimiento. En adición, la combinación de estos dos tipos de lixiviados ejerce un efecto sinérgico sobre la fisiología y producción de este vegetal.

Palabras clave: Agroindustrial; biomasa; radicular; rendimiento; residuos; vegetal.

\begin{abstract}
The objective of this study was to evaluate the effect of vermicompost leakage based on Vegetable Residues and Chicken Manure; Vegetable and Agroindustrial Residues, and the combination of the two leakages, in rates of 60 y $90 \mathrm{~L} \mathrm{ha}^{-1} \mathrm{on}$ the growth and pepper production. The study was carried out in Marconi and Wonder pepper varieties, in total seven treatments were established in three repetitions. The best response in fruit diameter in both evaluated varieties corresponded to the Vegetable and Agroindustrial Residues Leakage $90 \mathrm{~L} \mathrm{ha}^{-1}$ and Combined Leakage $90 \mathrm{~L} \mathrm{ha}^{-1}$ treatments, being 52 and $15 \%$ higher than the control, respectively. On the other hand, the longest fruit length was evidenced in the Combined Leakage $90 \mathrm{~L} \mathrm{ha}^{-1}$ treatment. Similarly, the root biomass increased in all treatments where leakage was applied. In production, defined by fruit weight, the outstanding treatments were Vegetable and Agroindustrial Residues Leakage $90 \mathrm{~L} \mathrm{ha}^{-1}$ and Combined Leakage $90 \mathrm{~L} \mathrm{ha}^{-1}$. Thus, we can conclude that the use of vermicompost leakage in rates of $90 \mathrm{~L} \mathrm{ha}^{-1}$ represents an alternative to enhance pepper yield. Furthermore, the combination of these two types of leakage caused a synergistic effect on the physiology and vegetal production.
\end{abstract}

Keywords: Agroindustrial; biomass; root; yield; residues; vegetable.

\section{INTRODUCCIÓN}

El pimiento (Capsicum annuum L.) es un vegetal que posee un alto contenido de calorías, fibra, vitaminas y antioxidantes, por lo que es altamente recomendado como un alimento que aporta en la salud humana. Estos atributos han hecho del pimiento uno de los productos infaltables en la comida de los ecuatorianos. Por ello, en el agro y en el mercado se experimenta una mayor demanda (1). De acuerdo con el III Censo Nacional Agropecuario el cultivo de pimiento en el Ecuador alcanza una superficie total de 956 ha. En todo el mundo se producen 34497,462 toneladas de pimiento cultivados en 1938,788 ha (2).

Con el incremento de la población mundial, la demanda del pimiento ha ido en aumento año tras año (3). En este sentido, la fertilización del cultivo es una de las prácticas más importantes, ya que proporciona los nutrimentos necesarios para obtener altos rendimientos, de calidad y cumplir con los requisitos que exige el mercado (4). Sin embargo, el uso inadecuado de fertilizantes químicos ha originado una disminución en el contenido de la materia orgánica y deterioro del suelo; además, representa altos costos para los productores siendo necesario incursionar en el uso de técnicas que permitan reducir los costos de producción, sean amigables con el medio ambiente y permitan conservar el recurso suelo (5).

Entre estos métodos alternativos para la fertilización se encuentra la aplicación de abonos orgánicos como: compost, estiércol, vermicompost etc. Los abonos orgánicos funcionan como un almacén de nutrientes para la planta. El contenido nutrimental de los abonos orgánicos es muy variable y depende de la especie animal, residuo que se emplee, método de compostaje, etc. (4).

Los abonos orgánicos son componentes clave de la fertilidad del suelo. Controlan las actividades de la rizósfera y regulan el crecimiento de las plantas. Las sustancias húmicas contenidas en estos compuestos orgánicos al ser aplicadas al 
suelo contribuyen sustancialmente a mejorar la calidad del suelo, efecto evidenciado por el incremento de biomasa de raíces y rendimiento (6). En este contexto es necesario evaluar el comportamiento agronómico de los cultivos frente a nuevos productos orgánicos como es el caso del vermicompost y sus lixiviados, que podrían convertirse en una opción de fertilización orgánica o bioestimulación de los cultivos que conserva el medio ambiente y su implementación resulta económica.

Dada la importancia económica que representa el cultivo de pimiento en el Ecuador y a la escasa información disponible relacionada al uso de fuentes orgánicas como es el caso de los lixiviados de vermicompost sobre el cultivo de pimiento, el objetivo de esta investigación fué evaluar la respuesta de dos variedades de pimiento a la aplicación de lixiviados orgánicos obtenidos a partir de la producción de vermicompost.

\section{MATERIALES Y METODOS}

Localización del experimento. El experimento se realizó en macetas de $3 \mathrm{~kg}$ de capacidad que fueron llenas con suelo tamizado. Todas las plantas se cultivaron en condiciones controladas de invernadero (temperatura de entre $25 \pm 2{ }^{\circ} \mathrm{C}$; humedad del aire promedio de 70\%) en el campus del Instituto Superior Tecnológico Enrique Noboa, en la ciudad de La Troncal, provincia del Cañar, Ecuador.

Características del suelo. Se recolectaron muestras de suelo a $20 \mathrm{~cm}$. de profundidad en una finca localizada entre las coordenadas $2^{\circ} 24^{\prime} 44,6^{\prime \prime} \mathrm{S}$ y $79^{\circ} 20^{\prime} 40,8^{\prime \prime} \mathrm{W}$ a una altura de $17 \mathrm{msnm}$, La Troncal, Ecuador. El suelo fué secado al aire libre y luego tamizado a través de un tamiz de $2 \mathrm{~mm}$ para su posterior homogenización. Según la clasificación Soil Taxonomy, el suelo corresponde a un Fluventic ustropepts, franco con textura media, presenta CIC $27 \mathrm{cmol} \mathrm{kg}^{-1}$; $\mathrm{MO} 2,9 \%$; $\mathrm{pH}$ 5,76; valores de Fósforo- Bray II de 14,8 ppm; Potasio disponible 0,35 $\mathrm{cmol} \mathrm{kg}^{-1}$. Las variables meteorológicas del sitio responden a valores de temperatura promedio anual de $26,7^{\circ} \mathrm{C}$, humedad relativa promedio $87 \%$ y precipitación anual de $1.811 \mathrm{~mm}(7)$

Lixiviados estudiados. La obtención de lixiviados de vermicompost, se realizó a partir de la producción de vermicompost

Obtención de vermicompost. Las proporciones de residuos para producir el vermicompost a partir del cual se recolectaron sus fluidos líquidos (lixiviados) para aplicación en el ensayo fueron:

LRVG con Mezcla de Residuos Vegetales (restos de podas de árboles y restos de vegetales) 70\% + Estiércol de Gallinaza 25\% + Aserrín 5\%; LRVA con Mezcla de Residuos Vegetales (restos de podas de árboles y restos de vegetales) 70\% + Residuos Agroindustriales (obtenidos de la industria azucarera a partir de caña de azúcar cachaza y bagazo) 30\%. En la tabla 1, se presentan algunas características de los residuos a partir de los cuales se produjo el lixiviado de vermicompost

Tabla 1. Características de los residuos empleados para producir vermicompost.

\begin{tabular}{ccccccc}
\hline Parámetros & Restos de podas de árboles & Residuos vegetales & Gallinaza & Aserrín & Cachaza & Bagazo \\
\hline Relación C/N & 47 & 37 & 7 & 108 & 35 & 104 \\
Tamaño de partícula mm & $5-20$ & $5-20$ & $5-10$ & $1-10$ & $>2$ & $10-20$ \\
\% Humedad & 30 & 45 & 22 & 25 & 67 & 36 \\
\hline
\end{tabular}

El proceso para la producción del vermicompost se realizó en campus del Instituto Superior Tecnológico Enrique Noboa, en la ciudad de La Troncal, provincia del Cañar, Ecuador. Las lombrices de la especie Eisenia sp fueron adquiridas mediante donación por el Departamento de Investigaciones de Campo del Ingenio Azucarero Agro azúcar - La Troncal. Se construyeron dos cajas de madera con medidas de 1.5 metros de longitud por 0.6 metros de ancho por 0.5 metros de altura en las cuales se colocaron los residuos:

Caja 1: LRVG con Mezcla de Residuos Vegetales (restos de podas de árboles y restos de vegetales) 70\% + Estiércol de Gallinaza 25\% + Aserrín 5\%;

Caja 2: LRVA con Mezcla de Residuos Vegetales (restos de podas de árboles y restos de vegetales) $70 \%+$ Residuos Agroindustriales (obtenidos de la industria azucarera a partir de caña de azúcar cachaza y bagazo) $30 \%$.

Al interior de las cajas se colocó plástico. Luego de 2 días del llenado de residuos en las cajas y de efectuarse riego hasta humedecer los residuos en rangos de 80\%, se efectuó la siembra de 0,5 kg de lombrices por cada $10 \mathrm{~kg}$ de residuos. 
Para el mantenimiento de las cajas o lombriceras se monitoreo humedad y temperatura. Durante todo el proceso de descomposición de los residuos la temperatura se procuró mantener entre 18 y $23{ }^{\circ} \mathrm{C}$, para controlar las subidas de temperatura se realizaba remociones y/o riegos. De tal manera se ejecutaron remociones cada cinco días y aplicaciones de riegos cada dos días manteniendo la humedad de las lombriceras entre 75 y $80 \%$. Después de 3 meses de haber iniciado el proceso de vermicompostaje se tomaron las muestras de lixiviado de vermicompost para aplicar en el ensayo. Algunas propiedades químicas de estos lixiviados se presentan a continuación: LRVG con valores de Materia orgánica 0.31\%; Nitrógeno 0,21 g 100g-1; Fósforo 0,03 g $100 \mathrm{~g}^{-1}$; Potasio 0,22 g $100 \mathrm{~g}^{-1}$ LRVA con valores de Materia orgánica 0.33\%; Nitrógeno 0,22 g $100 \mathrm{~g}^{-1}$; Fósforo 0,04 g 100 $\mathrm{g}^{-1}$; Potasio 0,22 g $100 \mathrm{~g}^{-1}$ (7).

Procedimiento experimental. Se estudiaron 2 variedades de pimiento: Marconi y Wonder. Ambas variedades caracterizadas por su madurez entre los 100-160 días después de la siembra (DDS). Variedad Marconi caracterizada por su aptitud de siembra para todo el año, su fruto es puntiagudo, pasando del color verde al rojo. Por otro lado, la variedad Wonder muy productiva con 15 a 20 toneladas por ha y resistente a muchos climas, se adapta entre 0 a 2100 msnm. Sus frutos maduran del verde al rojo brillante y tienen una carne gruesa, tierna y muy dulce. Es una planta mediana que mide entre 60 y $70 \mathrm{~cm}$.

Para el establecimiento del ensayo se procedió directamente sembrando las semillas en el suelo previamente tamizado y distribuido en macetas con medidas de $18 \mathrm{~cm}$ de ancho x $29 \mathrm{~cm}$ de alto. Cada maceta contenía $3 \mathrm{~kg}$ de suelo. Posterior a esto a los 30 DDS se fertilizó a todos los tratamientos incluyendo el control con una dosis de $200 \mathrm{~kg} \mathrm{~N} \mathrm{ha}^{-1} ; 80 \mathrm{Kg} \mathrm{P}_{2} \mathrm{O}_{5}$ ha-1; $300 \mathrm{~K}_{2} \mathrm{O} \mathrm{ha}^{-1}$, estos valores calculados para las cantidades de suelo con las que se trabajó en las macetas correspondieron a: 0,3 $\mathrm{g} \mathrm{N}$ maceta $^{-1} ; 0,12 \mathrm{~g} \mathrm{P}_{2} \mathrm{O}_{5}$ maceta $^{-1} ; 0,45 \mathrm{~g} \mathrm{~K}_{2} \mathrm{O}$ maceta $^{-1}$. El cultivo de pimiento extrae altas cantidades de nutriente $\mathrm{N}-\mathrm{P}_{2} \mathrm{O}_{5}-\mathrm{K}_{2} \mathrm{O}$ sobre todo en condiciones de invernadero, por esta razón se aplicó una dosis promedio a la establecida en invernaderos de la localidad en escala comercial.

Los tratamientos evaluados fueron: T1= Control (Sin lixiviado); T2= LRVG $60 \mathrm{~L} \mathrm{ha}^{-1}$; T3= LRVA 60 L ha ${ }^{-1}$; T4= Lixiviado combinado LC $60 \mathrm{~L} \mathrm{ha}^{-1}$; T5= LRVG $90 \mathrm{~L} \mathrm{ha}^{-1}$; T6= LRVA $90 \mathrm{~L} \mathrm{ha}^{-1}$; LC $90 \mathrm{~L} \mathrm{ha}^{-1}$. La aplicación de dosis de lixiviados de vermicompost de 60 y $90 \mathrm{~L} \mathrm{ha}^{-1}$ acorde a los tratamientos establecidos se realizó de forma fraccionada en 2 aplicaciones al 50\%, la primera aplicación a la edad de 60 DDS y la segunda a los 75 DDS. Estos valores calculados para las cantidades de suelo con las que se trabajó en las macetas correspondieron: Para la dosis de $60 \mathrm{~L} \mathrm{ha}^{-1}$ a 0,05 ml de lixiviado disuelto en $0.26 \mathrm{ml}$ de agua maceta ${ }^{-1}$; Para la dosis de $90 \mathrm{~L} \mathrm{ha}^{-1}$ a $0,14 \mathrm{ml}$ de lixiviado disuelto en $0.17 \mathrm{ml} \mathrm{de} \mathrm{agua} \mathrm{maceta}^{-1}$. Es decir, se aplicaron $0.3 \mathrm{ml}$ de solución lixiviado + agua por cada maceta mediante una jeringa. Realizándose 2 aplicaciones para completar la dosis establecida

La cosecha del ensayo se realizó cuando las plantas llegaron a la madurez fisiológica, esto correspondió a los 110 DDS para la variedad Marconi y 145 DDS para la variedad Wonder. Siete tratamientos fueron evaluados para dos variedades de pimiento. Se consideraron tres repeticiones por cada variedad de pimiento, se evaluaron dos plantas de pimiento por cada maceta. En total 42 unidades experimentales.

Parámetros estudiados. En las plantas de pimiento se registraron las siguientes variables respuestas: La altura de planta (AP) se la determinó en cm a los 75 días después de la siembra, registrando el dato desde el nivel del suelo hasta la yema terminal de la rama más alta. El diámetro del tallo (DT) fue registrado al nivel del suelo con un calibrador graduado en mm. La longitud del fruto (LF) se determinó en cm con un flexómetro, para lo cual se promedió la longitud de los frutos por planta. El diámetro del fruto (DF) se registró en cm con un calibrador graduado, para lo cual se promedió el diámetro de los frutos por planta. El peso del fruto (PF) se determinó en gramos con una balanza analítica de precisión, para lo cual se promedió el peso de todos los frutos por unidad experimental. Una vez realizada la primera cosecha se procedió al muestreo destructivo donde se extrajeron las raíces y área foliar para someterlos a secado a $65^{\circ} \mathrm{C}$ por 24 horas en una estufa y posteriormente obtener los pesos secos mediante una balanza analítica de precisión.

Análisis estadístico. El estudio fue conducido usando un diseño completamente al azar con 7 tratamientos y 3 repeticiones evaluando las variedades (Marconi, Wonder). Los datos fueron analizados usando el ANOVA en una vía, la normalidad fue verificada usando el test Shapiro-Wilks y la separación de medias a partir de la prueba Duncan test $(p<0.05)$ usando el software InfoStat versión 2016e.

\section{RESULTADOS}

Variables evaluadas durante el desarrollo. En la tabla 2, se presenta los resultados obtenidos en las variables de altura de planta, número de hojas y diámetro de tallo. 
Tabla 2. Valores de las características fenológicas evaluadas durante el desarrollo del pimiento bajo diferentes dosis de lixiviados de vermicompost.

\begin{tabular}{|c|c|c|c|c|c|c|}
\hline \multirow{2}{*}{ Tratamientos } & \multicolumn{2}{|c|}{ Altura de planta $(\mathrm{cm})$} & \multicolumn{2}{|c|}{ Número de hojas } & \multicolumn{2}{|c|}{ Diámetro de tallo (mm) } \\
\hline & Marconi & Wonder & Marconi & Wonder & Marconi & Wonder \\
\hline 1.- Control & $18.7 \pm 0.6 \mathrm{a}$ & $15.8 \pm 0.9 \mathrm{a}$ & $8.7 \pm 0.6$ bc & $7.8 \pm 1.2 \mathrm{bc}$ & $4.5 \pm 0.1 \mathrm{a}$ & $4 . .0 \pm 0.1 \mathrm{a}$ \\
\hline 2.-LRVG. 60 & $26.5 \pm 4.8 \mathrm{a}$ & $18.1 \pm 2.4 \mathrm{a}$ & $11.0 \pm 1.0 \mathrm{bc}$ & $7.2 \pm 0.7 \mathrm{bc}$ & $4.3 \pm 0.0 \mathrm{a}$ & $4.8 \pm 0.0 \mathrm{a}$ \\
\hline 3.- LRVA. 60 & $18.3 \pm 4.5 \mathrm{a}$ & $16.0 \pm 0.8 \mathrm{a}$ & $8.5 \pm 0.6 \mathrm{c}$ & $6.2 \pm 1.3 \mathrm{c}$ & $4.5 \pm 0.0 \mathrm{a}$ & $4.7 \pm 0.0 \mathrm{a}$ \\
\hline 4.-LC.60 & $25.2 \pm 2.8 \mathrm{a}$ & $18.2 \pm 1.1 \mathrm{a}$ & $11.5 \pm 1.3 \mathrm{ab}$ & $7.5 \pm 0.2 \mathrm{bc}$ & $5.0 \pm 0.0 \mathrm{a}$ & $4.8 \pm 0.0 \mathrm{a}$ \\
\hline 5.-LRVG.90 & $25.6 \pm 1.4 \mathrm{a}$ & $14.7 \pm 0.7 \mathrm{a}$ & $12.8 \pm 0.2 \mathrm{a}$ & $6.5 \pm 0.9 \mathrm{c}$ & $4.5 \pm 0.1 \mathrm{a}$ & $4.3 \pm 0.0 \mathrm{a}$ \\
\hline 6.-LRVA.90 & $25.0 \pm 0.4 \mathrm{a}$ & $18.9 \pm 0.6 \mathrm{a}$ & $11.2 \pm 0.8 \mathrm{abc}$ & $10.7 \pm 1.0 \mathrm{a}$ & $4.3 \pm 0.0 \mathrm{a}$ & $4.3 \pm 0.0 \mathrm{a}$ \\
\hline 7.-LC.90 & $25.0 \pm 2.9 \mathrm{a}$ & $17.1 \pm 1.8 \mathrm{a}$ & $9.7 \pm 1.0 \mathrm{bc}$ & $10.0 \pm 0.4 \mathrm{ab}$ & $4.3 \pm 0.0 \mathrm{a}$ & $4.2 \pm 0.0 \mathrm{a}$ \\
\hline (p-valor) & 0.31 & 0.33 & 0.03 & 0.02 & 0.85 & 0.51 \\
\hline
\end{tabular}

Valores son medias de tres réplicas. Error estándar: \pm . Valores seguidos por la misma letra en la columna indican que no existen diferencias significativas $(\mathrm{p}<0.05)$ para el test Duncan. Control= Sin lixiviado; LRVG.60= Lixiviado de residuos vegetales y gallinaza $60 \mathrm{~L}$ ha-1; LRVA.60= Lixiviado de residuos vegetales y agroindustriales $60 \mathrm{~L}$ ha-1; LC.60= Lixiviado combinado $60 \mathrm{~L}$ ha-1; LRVG.90= Lixiviado de residuos vegetales y gallinaza $90 \mathrm{~L}$ ha-1; LRVA.90= Lixiviado de residuos vegetales y agroindustriales 90 L ha-1; LC.90= Lixiviado combinado 90 L ha-1;

En la altura de planta expresa en $\mathrm{cm}$. y diámetro de tallo expresado en $\mathrm{mm}$, no se encontró diferencias significativas $(\mathrm{P}<$ $0.05)$ entre los tratamientos.

Respecto a la variable de número de hojas por planta en la variedad Marconi el tratamiento LRVG 90 L ha-1 presentó valor de 12.8 hojas por planta con diferencias significativas $(\mathrm{p}<0.05)$ y superando al control. Así mismo, en la variedad Wonder el mejor tratamiento con diferencias significativas $(\mathrm{p}<0.05)$ respecto al control, fue el LRVA $90 \mathrm{~L} \mathrm{ha}^{-1}$, con 10.7 hojas por planta.

Variables evaluadas durante la cosecha. En la tabla 3, se exponen los resultados de las variables de diámetro de fruto y longitud de fruto expresadas en $\mathrm{cm}$.

Tabla 3. Valores de las características fenológicas evaluadas durante la cosecha del pimiento bajo diferentes dosis de lixiviados de vermicompost.

\begin{tabular}{|c|c|c|c|c|}
\hline \multirow{2}{*}{ Tratamientos } & \multicolumn{2}{|c|}{ Diámetro del fruto $(\mathrm{cm})$} & \multicolumn{2}{|c|}{ Longitud del fruto $(\mathrm{cm})$} \\
\hline & Marconi & Wonder & Marconi & Wonder \\
\hline 1.- Control & $2.5 \pm 0.1 \mathrm{c}$ & $3.9 \pm 0.1 \mathrm{~cd}$ & $7.5 \pm 0.3 \mathrm{~d}$ & $4.1 \pm 0.1 \mathrm{~d}$ \\
\hline 2.-LRVG. 60 & $3.0 \pm 0.3 \mathrm{abc}$ & $3.7 \pm 0.3 \mathrm{~d}$ & $9.2 \pm 0.1 \mathrm{~b}$ & $4.2 \pm 0.2 \mathrm{~d}$ \\
\hline 3.- LRVA. 60 & $3.1 \pm 0.3 \mathrm{abc}$ & $4.5 \pm 0.1 \mathrm{~b}$ & $7.1 \pm 0.4 \mathrm{~d}$ & $5.2 \pm 0.2 \mathrm{c}$ \\
\hline 4.-LC.60 & $2.8 \pm 0.3 \mathrm{bc}$ & $4.0 \pm .0 .2 \mathrm{~cd}$ & $9.2 \pm 0.0 \mathrm{~b}$ & $5.2 \pm 0.5 b c$ \\
\hline 5.-LRVG.90 & $3.3 \pm 0.1 \mathrm{abc}$ & $4.4 \pm 0.0 \mathrm{bc}$ & $8.9 \pm 0.1 \mathrm{bc}$ & $5.3 \pm 0.1 \mathrm{ab}$ \\
\hline 6.-LRVA.90 & $3.5 \pm 0.1 \mathrm{ab}$ & $4.5 \pm 0.1 \mathrm{~b}$ & $8.0 \pm 0.8 \mathrm{~cd}$ & $4.5 \pm 0.1 \mathrm{~cd}$ \\
\hline 7.-LC.90 & $3.8 \pm 0.3 \mathrm{a}$ & $5.1 \pm 0.1 \mathrm{a}$ & $11.6 \pm 0.1 \mathrm{a}$ & $5.9 \pm 0.1 \mathrm{a}$ \\
\hline (p-valor) & 0.001 & 0.001 & 0.001 & 0.001 \\
\hline
\end{tabular}

Valores son medias de tres réplicas. Error estándar: \pm . Valores seguidos por la misma letra en la columna indican que no existen diferencias significativas $(\mathrm{p}<0.05)$ para el test Duncan. Control= Sin lixiviado; LRVG.60= Lixiviado de residuos vegetales y gallinaza $60 \mathrm{~L}$ ha-1; LRVA.60= Lixiviado de residuos vegetales y agroindustriales $60 \mathrm{~L}$ ha-1; LC.60= Lixiviado combinado $60 \mathrm{~L}$ ha-1; LRVG.90= Lixiviado de residuos vegetales y gallinaza $90 \mathrm{~L}$ ha-1; LRVA.90= Lixiviado de residuos vegetales y agroindustriales 90 L ha-1; LC.90= Lixiviado combinado 90 L ha-1;

En el diámetro de fruto podemos observar que, los tratamientos con diferencias significativas $(\mathrm{p}<0.05)$ que superaron al control fueron LC $90 \mathrm{~L} \mathrm{ha}^{-1}$; LRVA $90 \mathrm{~L} \mathrm{ha}^{-1}$ y LRVA $60 \mathrm{~L} \mathrm{ha}^{-1}$. De esta manera en la variedad Marconi los valores de este parámetro se ubicaron en 3.8 y $3.5 \mathrm{~cm}$, respectivamente. Mientras que, en la variedad Wonder los tratamientos que provocaron diferencias significativas $(\mathrm{p}<0.05)$ respecto al control fueron LC $90 \mathrm{~L} \mathrm{ha}^{-1}$; LRVA $90 \mathrm{~L} \mathrm{ha}^{-1}$; LRVA $60 \mathrm{~L} \mathrm{ha}^{-1}$ con valores de 5.1; 4.5 y $4.5 \mathrm{~cm}$ de diámetro de fruto, respectivamente. 
Por otro lado, en la longitud del fruto, en la variedad Marconi los tratamientos que superaron a control mostrando diferencias significativas (p<0.05) fueron: LC $90 \mathrm{~L} \mathrm{ha}^{-1}$, LC $60 \mathrm{~L} \mathrm{ha}^{-1}$ y LRVG $60 \mathrm{~L} \mathrm{ha}^{-1}$ con 11.6; 9.2 y $9.2 \mathrm{~cm}$ de longitud de fruto, respectivamente. Mientras que, en la variedad Wonder los tratamientos con diferencias significativas (p<0.05) y superiores al control fueron LC $90 \mathrm{~L} \mathrm{ha}^{-1}$ con valores de 5.9 y LRVG $90 \mathrm{~L} \mathrm{ha}^{-1}$ con $5.3 \mathrm{~cm}$ de longitud del fruto.

La tabla 4, muestra los resultados obtenidos en peso (gramos) de biomasa foliar y radicular.

Tabla 4. Influencia de diferentes dosis de lixiviados de vermicompost sobre la biomasa radicular y foliar del pimiento.

\begin{tabular}{|c|c|c|c|c|}
\hline \multirow{2}{*}{ Tratamientos } & \multicolumn{2}{|c|}{ Biomasa foliar (g planta $^{-1}$ ) } & \multicolumn{2}{|c|}{ Biomasa radicular (g planta-1) $^{-1}$ ) } \\
\hline & Marconi & Wonder & Marconi & Wonder \\
\hline 1.- Control & $6.43 \pm 0.9 \mathrm{c}$ & $5.0 \pm 0.1 \mathrm{c}$ & $4.4 \pm 0.6 \mathrm{~b}$ & $3.7 \pm 0.2 \mathrm{c}$ \\
\hline 2.-LRVG. 60 & $11.0 \pm 0.0 \mathrm{abc}$ & $4.5 \pm 0.3 \mathrm{ab}$ & $8.1 \pm 0.2 \mathrm{a}$ & $6.7 \pm 0.4 \mathrm{ab}$ \\
\hline 3.- LRVA. 60 & $6.6 \pm 0.6 \mathrm{c}$ & $8.2 \pm 0.1 \mathrm{a}$ & $7.4 \pm 0.2 \mathrm{a}$ & $7.0 \pm 0.3 \mathrm{ab}$ \\
\hline 4.-LC.60 & $9.6 \pm 0.9 \mathrm{ab}$ & $6.4 \pm 0.4 \mathrm{~b}$ & $7.5 \pm 0.7 a$ & $6.0 \pm 0.4 \mathrm{~b}$ \\
\hline 5.-LRVG.90 & $9.0 \pm 0.4 \mathrm{~b}$ & $8.7 \pm 0.2 \mathrm{a}$ & $7.4 \pm 0.3 \mathrm{a}$ & $6.8 \pm 0.2 \mathrm{ab}$ \\
\hline 6.-LRVA.90 & $11.9 \pm 1.1 \mathrm{a}$ & $8.4 \pm 0.2 \mathrm{a}$ & $7.5 \pm 0.1 \mathrm{a}$ & $6.8 \pm 0.7 \mathrm{ab}$ \\
\hline 7.-LC.90 & $11.1 \pm 0.8 \mathrm{ab}$ & $7.9 \pm 0.9 \mathrm{a}$ & $7.1 \pm 0.1 \mathrm{a}$ & $7.7 \pm 0.1 \mathrm{a}$ \\
\hline (p-valor) & 0.001 & 0.0003 & 0.0002 & 0.0001 \\
\hline
\end{tabular}

Valores son medias de tres réplicas. Error estándar: \pm . Valores seguidos por la misma letra en la columna indican que no existen diferencias significativas $(\mathrm{p}<0,05)$ para el test Duncan. Control= Sin lixiviado; LRVG.60= Lixiviado de residuos vegetales y gallinaza $60 \mathrm{~L}$ ha-1; LRVA.60= Lixiviado de residuos vegetales y agroindustriales 60 L ha-1; LC.60= Lixiviado combinado $60 \mathrm{~L}$ ha-1; LRVG.90= Lixiviado de residuos vegetales y gallinaza 90 L ha-1; LRVA.90= Lixiviado de residuos vegetales y agroindustriales 90 L ha-1; LC.90= Lixiviado combinado 90 L ha-1;

Al analizar la biomasa foliar, podemos ratificar que existe una respuesta positiva de la planta hacia las dosis incrementales de lixiviados, de esta manera en la variedad Marconi los tratamientos LRVA $90 \mathrm{~L} \mathrm{ha}^{-1}$; LC $90 \mathrm{~L} \mathrm{ha}^{-1}$, LRVG $90 \mathrm{~L}^{-1}$ y LC 60 $\mathrm{L} \mathrm{ha}^{-1}$ con $11.9 ; 11,1 ; 9,6 ; 9.0 \mathrm{~g}$ de biomasa foliar planta ${ }^{-1}$, respectivamente, sobresalieron al ser comparadas al control mostrando diferencias significativas $(\mathrm{p}<0.05)$. Comportamiento similar en la variedad Wonder, donde los tratamientos que destacaron fueron LRVG $90 \mathrm{~L} \mathrm{ha}^{-1}$; LRVA $90 \mathrm{~L} \mathrm{ha}^{-1}$; LRVA $60 \mathrm{~L} \mathrm{ha}^{-1}$; LC $90 \mathrm{~L} \mathrm{ha}^{-1}$, exhibiendo valores de 8,7; 8,4; 8,2; 7, $9 \mathrm{~g}$ de biomasa foliar planta ${ }^{-1}$.

En ambas variedades de pimiento, la biomasa radicular expresada en gramos de peso mostró diferencias significativas $(\mathrm{p}<0.05)$, donde todos los tratamientos aplicados con lixiviados mostraron ser superiores al control en esta variable. Cabe resaltar que en la variedad Wonder destacó el tratamiento LC $90 \mathrm{~L} \mathrm{ha}^{-1}$

Finalmente, en la figura 1, expone el peso del fruto expresado en gramos, donde la variedad Marconi con los tratamientos LRVA $90 \mathrm{~L} \mathrm{ha}^{-1}$ y LC $90 \mathrm{~L} \mathrm{ha}^{-1}$ alcanzó los mayores valores, que superaron a control con diferencias significativas $(\mathrm{p}<0.05)$, arrojaron valores de peso de 28.2 y $27.5 \mathrm{~g}$, respectivamente. Por otro lado, en la variedad Wonder superaron significativamente al control los siguientes tratamientos: LC $90 \mathrm{~L} \mathrm{ha}^{-1}$ con $33.2 \mathrm{~g}$ y los tratamientos LRVE 90 L ha-1 y LRVA $90 \mathrm{~L} \mathrm{ha}^{-1}$ con 28.7 y $28.3 \mathrm{~g}$, respectivamente. 


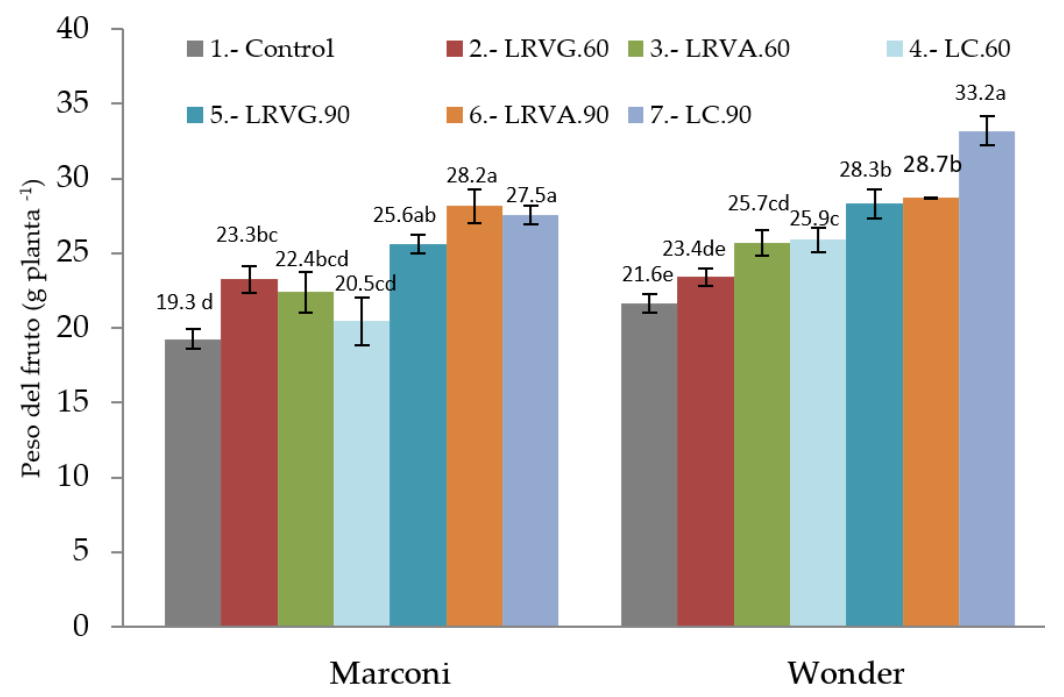

Figura 1. Influencia de diferentes dosis de lixiviados de vermicompost sobre el rendimiento del pimiento (g planta-1 $)$.

Valores son medias de tres réplicas. Error estándar: \pm . Valores seguidos por la misma letra en la columna indican que no existen diferencias significativas $(\mathrm{p}<0,05)$ para el test Duncan. Control= Sin lixiviado; LRVG.60= Lixiviado de residuos vegetales y gallinaza 60 L ha-1; LRVA.60= Lixiviado de residuos vegetales y agroindustriales $60 \mathrm{~L}$ ha-1; LC. $60=$ Lixiviado combinado $60 \mathrm{~L}$ ha-1; LRVG.90= Lixiviado de residuos vegetales y gallinaza 90 L ha-1; LRVA.90= Lixiviado de residuos vegetales y agroindustriales $90 \mathrm{~L}$ ha-1; LC.90= Lixiviado combinado $90 \mathrm{~L}$ ha-1;

\section{DISCUSION}

Según investigaciones de Acevedo et al (6) en cultivo de tomate donde se aplicaron abonos orgánicos se encontró mayor diámetro y altura de planta. En contraste con estos estudios, Rodríguez et al. (8) y Rodríguez et al (9) no encontraron respuesta significativa en las variables mencionadas, estos últimos hallazgos se alinean con los resultados expuestos en el presente estudio. Cabe mencionar que en nuestro trabajo se midió altura de planta y diámetro de tallo a los 75 DDS es posible que a esta edad los componentes del lixiviado de vermicompost aún no ejerzan efecto visible sobre estas variables evaluadas en las plantas.

Sin embargo, la variable correspondiente al número de hojas por planta mostró diferencia estadística significativa respecto al control, para el caso de la variedad Marconi con el tratamiento LRVG $90 \mathrm{~L} \mathrm{ha}^{-1}$, mientras que para la variedad Wonder con el tratamiento LRVA $90 \mathrm{~L} \mathrm{ha}^{-1}$, estos resultados son consistentes con los hallados por Vera et al. (10) quienes encontraron mayor vigor en las plantas de pimiento que se aplicaron con biol de gallinaza. Esta situación se puede asociar con la calidad nutricional de la gallinaza (4). Los efectos benéficos se atribuyen al hecho de que el vermicompost y sus derivados contienen aminoácidos, vitaminas, ácidos húmicos, ácidos fúlvicos y sustancias reguladoras del crecimiento (11). La capacidad que poseen los materiales vermicompostados de liberar lentamente nutrientes cuando son aplicados al suelo, de forma que sean accesibles a las plantas cuando éstas los necesitan, ha sido señalada por Arancon et al (12).

En las variables de diámetro y longitud del fruto, encontramos respuesta superior al control en los tratamientos LC $90 \mathrm{~L}$ ha $^{-1}$; LC $60 \mathrm{~L} \mathrm{ha}^{-1}$ y LRVG $60 \mathrm{~L} \mathrm{ha}^{-1}$, resultados concordantes con los reportados por Ikeh et al (13), quienes encontraron mayor longitud y diámetro del fruto con dosis de $8 \mathrm{t}$ ha de estiércol avícola, en contraste al tratamiento testigo. El efecto positivo del abono de aves de corral sobre los frutos en la planta de pimiento fue plasmado en los resultados también demostrados por Alabi (14). Sin embargo, se puede evidenciar que los lixiviados de vermicompost aplicados de manera individual no resultan tan efectivos como al aplicarlos combinados, al parecer existe una sinergia ente los componentes, esto puede explicarse ya que a pesar de que el vermicompost fué indicado por numerosos autores como una fuente de macronutrientes para la planta; el efecto del humus sobre la fisiología de la planta dependerá del tiempo de procesamiento y la madurez de este (15). Aunque el vermicompost y sus lixiviados también aporta macronutrientes al suelo, varios estudios evidencian que el uso de estos productos no provee el suficiente nitrógeno para las diferentes funciones metabólicas de las plantas, como los fertilizantes químicos convencionales, debido a su alta resistencia a la degradación y la baja velocidad de disponibilidad y mineralización de los nutrientes a partir de los abonos orgánicos. Esto pudo influir en los resultados obtenidos de manera individual, sin embargo, al combinar los lixiviados y en mayor dosis se incrementa la efectividad del producto. Este comportamiento puede explicarse debido a que las sustancias húmicas contenidas en los lixiviados y una vez aplicadas en las plantas, estimulan la tasa de fotosíntesis y reparación de las plantas debido a la presencia de fitohormonas como auxinas y giberelinas que están directamente involucradas en 
varios procesos bioquímicos al interior de las células vegetales y actividades enzimáticas implicadas en el metabolismo de los carbohidratos (16)

$\mathrm{Al}$ analizar el comportamiento de la biomasa foliar, podemos ratificar que existe una respuesta positiva de la planta hacia las dosis incrementales de lixiviados, de esta manera los tratamientos sobresalientes fueron LRVA $90 \mathrm{~L} \mathrm{ha}^{-1}$; LC $90 \mathrm{~L} \mathrm{ha}^{-1}$, LRVG $90 \mathrm{~L} \mathrm{ha}^{-1}$.

La biomasa radicular se vio influenciada positivamente en todos los tratamientos donde se aplicó el lixiviado, con diferencia significativa respecto al control. Esta respuesta, puede explicarse debido a la estimulación significativa inducida por los lixiviados sobre la biomasa radicular, situación probablemente influenciada por la presencia de elementos minerales, aminoácidos, proteínas y microorganismos presentes en el lixiviado, que estimulan la formación de células corticales y epidérmicas de la raíz aumentando la elongación y emergencia radicular y la actividad de ATP en las membranas de las raíces (17). En este sentido, Hernández et al (18), reportaron resultados similares en un cultivo de arroz, donde los lixiviados húmicos incrementaron la emisión de raíces y pelos radicales en fase de germinación en cultivo de arroz. Estos efectos se atribuyen a la composición de los lixiviados, que aportan sustancias reguladoras de crecimiento y ácidos húmicos que son responsables del crecimiento de las plantas (19).

El peso del fruto expresado en gramos con los tratamientos LRVA $90 \mathrm{~L} \mathrm{ha}^{-1}$; LC $90 \mathrm{~L} \mathrm{ha}^{-1}$ y LRVE 90 L ha-1 alcanzó los mayores valores que superaron a control. Dosis altas en nuestro caso la de $90 \mathrm{~L} \mathrm{ha}^{-1}$ garantizó incrementos en la producción, nuestros resultados son concordantes con los reportados por Reyes et al (20), quienes aplicaron altas dosis de lixiviado de lombriz en tomate y obtuvieron mayores rendimientos. Los efectos positivos de la aplicación de cualquier materia orgánica, están dados porque las oligosacarinas se desprenden de la pared celular por acción enzimática, lo que regula la tasa de crecimiento; de ahí que se consideran reguladores del crecimiento de la planta (17). Este proceso sucede inicialmente con el incremento de actividad en la rizósfora evidenciado en el presente estudio por aumento de biomasa radicular (Tabla 3), así la planta incrementa su eficiencia en la absorción de nutrientes, adicionalmente puede aprovechar los compuestos húmicos lábiles contenidos en los lixiviados, potenciando el intercambio gaseoso y fomentando la concentración de azucares llegando a incrementar su biomasa foliar biomasa foliar y consiguiente producción creciente.

En conclusión, la mejor respuesta en diámetro del fruto en ambas variedades evaluadas correspondió a los tratamientos LRVA $90 \mathrm{~L} \mathrm{ha}^{-1}$ y LC $90 \mathrm{~L} \mathrm{ha}^{-1}$ siendo superiores al control con valores de entre 52 y 15\%. Por otro lado, en la variable de longitud del fruto el tratamiento que sobresalió fue el LC $90 \mathrm{~L} \mathrm{ha}^{-1}$ con incrementos del orden de 55 y $44 \%$ vs control. Finalmente, los mejores rendimientos de peso del fruto se obtuvieron con los tratamientos LRVA $90 \mathrm{~L} \mathrm{ha}^{-1}$ y LC $90 \mathrm{~L}^{-}$ ha $^{-1}$. Estos resultados sugieren que es factible mejorar la productividad el cultivo de pimiento mediante la aplicación de estos lixiviados de vermicompost en dosis de $90 \mathrm{~L} \mathrm{ha}^{-1}$, sin embargo, aún hace falta investigar al detalle los procesos bioquímicos que suceden en el vegetal y desencadenan mejoras notables en el desarrollo y producción de los cultivos. Además, es un imperativo seguir evaluando el efecto de lixiviados de vermicompost procedentes de diferentes residuos.

\section{Conflictos de interes}

Declaramos no tener conflictos de interés en el trabajo presentado

\section{REFERENCIAS}

1. Matsufuji H, Ishikawa K, Nunomura O, Chino M, Takeda M. Anti-oxidant content of different coloured sweet peppers, white, green, yellow, orange and red (Capsicum annuum L.). Int J Food Sci Technol. 2007; 42(12):1482-1488. https:// ifst.onlinelibrary.wiley.com/doi/abs/10.1111/j.1365-2621.2006.01368.x

2. FAO. FAOSTAT Anuario estadístico de la FAO 2017. Organización de las Naciones Unidas para la Alimentación y la Agricultura2018. http://www.fao.org/3/b-i6407s.pdf

3. Malik AA, Chattoo MA, Sheemar G, Rashid R. Growth, yield and fruit quality of sweet pepper hybrid SH-SP-5 (Capsicum annuum L.) as affected by integration of inorganic fertilizers and organic manures (FYM). J Agric Technol. 2011; 7(4):1037-1048. http://www.ijat-aatsea.com/pdf/July v7 n4 11/16\%20IJAT2011 Malik R.pdf

4. Duarte RM, Contreras RLG, Contreras FR. Respuesta de la aplicación de estiércol y fertilizantes sobre el rendimiento y calidad del chile jalapeño. Biotecnia. 2012; 14(3):32-38. https://biotecnia.unison.mx/index.php/biotecnia/ article/view/127/120 
5. Adekiya AO, Agbede TM. Growth and yield of tomato (Lycopersicon esculentum Mill) as influenced by poultry manure and NPK fertilizer. Emir J Food Agric. 2009; 21(1):10-20. https://doi.org/10.9755/ejfa.v21i1.5154

6. Ciarkowska, K.; Solek-Podwika, K.; Filipek-Mazur, B.; Tabak, M. Comparative effects of lignite-derived humic acids and FYM on soil properties and vegetable yield. Geoderma 2017, 303(1):85-92. https://doi.org/10.1016/j. geoderma.2017.05.022

7. CINCAE. Centro de Investigaciones de la Caña de azúcar. Guayaquil-Ecuador. Informe Anual 2016. https://cincae. org/wp-content/uploads/2013/04/Informe-Anual-2016.pdf

8. Rodríguez-Dimas N, Cano-Ríos P, Favela-Chávez E, Figueroa-Viramontes U, De Paul-Álvarez V, Palomo-Gil A, et al. Vermicomposta como alternativa orgánica en la producción de tomate en invernadero. Rev Chapingo Ser Hortic. 2007; 13(2):185-192. https://www.redalyc.org/articulo.oa?id=60913280011

9. Rodríguez Dimas N, Cano Ríos P, Figueroa Viramontes U, Favela Chávez E, Moreno Reséndez A, Márquez Hernández C, et al. Uso de abonos orgánicos en la producción de tomate en invernadero. Terra Latinoam. 2009; 27(4):319-327. http://www.scielo.org.mx/scielo.php?pid=S0187-57792009000400006\&script=sci_abstract\&tlng=en

10. Vera EFM, García GAC, Chávez JEC, Villacorta HS, Vidal LRL. Efecto del biol bovino y avícola en la producción de pimiento dulce (Capsicum annum L.). Rev ESPAMCIENCIA. 2016; 7(1):15-21. http://190.15.136.171:4871/index. $\mathrm{php} /$ Revista_ESPAMCIENCIA/article/view/157

11. Aremu AO, Stirk WA, Kulkarni MG, Tarkowská D, Turečková V, Gruz J, et al. Evidence of phytohormones and phenolic acids variability in garden-waste-derived vermicompost leachate, a well-known plant growth stimulant. Plant Growth Regul. 2015; 75(2):483-492. https://pubag.nal.usda.gov/catalog/1212793

12. Arancon NQ Edwards CA, Bierman P. Influences of vermicomposts on field strawberries: Part 2. Effects on soil microbiological and chemical properties. Bioresour Technol. 2006; 97(6):831-840. http://citeseerx.ist.psu.edu/ viewdoc/download?doi=10.1.1.599.1172\&rep=rep1\&type=pdf

13. Ikeh AO, Ndaeyo NU, Uduak IG, Iwo GA, Ugbe LA, Udoh EI, et al. Growth and yield responses of pepper (Capsicum frutescens L.) to varied poultry manure rates in Uyo, southeastern Nigeria. J Agric Biol Sci. 2012; 7(9):735-742. https://www.cabdirect.org/cabdirect/abstract/20123393096

14. Alabi DA. Effects of fertilizer phosphorus and poultry droppings treatments on growth and nutrient components of pepper (Capsicum annuum L). Afr J Biotechnol. 2006; 5(8):671-677. https://www.ajol.info/index.php/ajb/article/ view/138122

15. Campitelli P, Ceppi S. Chemical, physical and biological compost and vermicompost characterization: A chemometric study. Chemom Intell Lab Syst. 2008; 90(1):64-71. https://www.infona.pl/resource/bwmeta1.element.elsevier458c745b-1c0c-3e12-bcdf-02c73baf2a5a

16. Nardi S, Pizzeghello D, Muscolo A, Vianello A. Physiological effects of humic substances on higher plants. Soil Biol Biochem. 2002; 34(11):1527-1536. https://www.bioag.com/wp-content/uploads/2018/05/Physiological effects of humic substances on higher plants.pdf

17. Canellas LP, Olivares FL, Okorokova-Façanha AL, Façanha AR. Humic acids isolated from earthworm compost enhance root elongation, lateral root emergence, and plasma membrane $\mathrm{H}+$-ATPase activity in maize roots. Plant Physiol. 2002; 130(4):1951-1957. https://europepmc.org/article/med/12481077

18. Hernández R, García A, Portuondo L, Muñiz S, Berbara R, Izquierdo F. Protección antioxidativa de los ácidos húmicos extraídos de vermicompost en arroz (Oryza sativa L.) var. IACuba30. Rev Protección Veg. 2012; 27(2):102-110. http://scielo.sld.cu/scielo.php?script=sci_arttext\&pid=S1010-27522012000200006

19. Alcívar M, Zurita-Silva A, Sandoval M, Muñoz C, Schoebitz M. Reclamation of saline-sodic soils with combined amendments: impact on quinoa performance and biological soil quality. Sustainability. 2018; 10(9):3083. https:// www.mdpi.com/2071-1050/10/9/3083

20. Reyes G, Cortés-Sánchez J. Intensidad En El Uso De Fertilizantes En América Latina Y El Caribe (2006-2012). Bioagro. 2017; 29(1):45-52. http://ve.scielo.org/scielo.php?script=sci arttext\&pid=S1316-33612017000100005\&lng=es\& $\underline{\mathrm{nrm}=\mathrm{iso}>}$ 